\title{
Transcription of the rat testis-specific Rtdpoz-TI and -T2 retrogenes during embryo development: co-transcription and frequent exonisation of transposable element sequences Chiu-Jung Huang1,2, Wan-Yi Lin ${ }^{3}$, Che-Ming Chang ${ }^{2}$ and Kong- Bung Choo*3,4,5
}

\begin{abstract}
Address: ${ }^{1}$ Department of Animal Science, School of Agriculture, Chinese Culture University, Yang-Ming-Shan, Taipei, Taiwan, ${ }^{2}$ Graduate Institute of Biotechnology, School of Agriculture, Chinese Culture University, Yang-Ming-Shan, Taipei, Taiwan, ${ }^{3}$ Department of Medical Research and Education, Taipei Veterans General Hospital, Shipai, Taipei, Taiwan, ${ }^{4}$ Graduate Program, Department of Biotechnology and Laboratory Science in Medicine, School of Biomedical Science and Engineering, National Yang Ming University, Shipai, Taipei, Taiwan and ${ }^{5}$ Institute of Clinical Medicine, School of Medicine, National Yang Ming University, Shipai, Taipei, Taiwan

Email: Chiu-Jung Huang - hqr2@faculty.pccu.edu.tw; Wan-Yi Lin - wylin@vghtpe.gov.tw; Che-Ming Chang - cgm44a@yahoo.com.tw; KongBung Choo* - kbchu@vghtpe.gov.tw

* Corresponding author
\end{abstract}

Published: 25 July 2009

BMC Molecular Biology 2009, 10:74 doi:10.1 186/147/-2199-10-74
Received: 20 March 2009

Accepted: 25 July 2009

This article is available from: http://www.biomedcentral.com/147/-2199/10/74

(c) 2009 Huang et al; licensee BioMed Central Ltd.

This is an Open Access article distributed under the terms of the Creative Commons Attribution License (http://creativecommons.org/licenses/by/2.0), which permits unrestricted use, distribution, and reproduction in any medium, provided the original work is properly cited.

\begin{abstract}
Background: Retrotransposition is an important evolutionary force for the creation of new and potentially functional intronless genes which are collectively called retrogenes. Many retrogenes are expressed in the testis and the gene products have been shown to actively participate in spermatogenesis and other unique functions of the male germline. We have previously reported a cluster of retrogenes in the rat genome that encode putative TRAF- and POZ-domain proteins. Two of the genes, Rtdpoz-TI and -T2 (abbreviated as $T I$ and T2), have further been shown to be expressed specifically in the rat testis.

Results: We show here that the $T I$ and $T 2$ genes are also expressed in the rat embryo up to days 16-17 of development when the genes are silenced until being re-activated in the adult testis. On database interrogation, we find that some $T I / T 2$ exons are chromosomally duplicated as cassettes of 2 or 3 exons consistent with retro-duplication. The embryonic TI/T2 transcripts, characterised by RT-PCR-cloning and rapid amplification of CDNA ends, are further found to have acquired one or more noncoding exons in the $5^{\prime}$-untranslated region ( $\left.5^{\prime}-U T R\right)$. Most importantly, the TI/T2 locus is embedded within a dense field of relics of transposable element (TE) derived mainly from LINEI and ERV sequences, and the TE sequences are frequently exonised through alternative splicing to form the $5^{\prime}$-UTR sequences of the TI/T2 transcripts. In a case of $T I$ transcript, the 3 '-end is extended into and terminated within an $\mathrm{LI}$ sequence. Since the two genes share a common exon $\mathrm{I}$ and are, therefore, regulated by a single promoter, a T2-to- $\mathrm{TI}$ co-transcription model is proposed. We further demonstrate that the exonised 5'-UTR TE sequences could lead to the creation of upstream open reading frames resulting in translational repression.
\end{abstract}

Conclusion: Exonisation of TE sequences is a frequent event in the transcription of retrogenes during embryonic development and in the testis and may contribute to post-transcriptional regulation of expression of retrogenes. 


\section{Background}

Retrotransposition is an important evolutionary driving force for the creation of new genes with novel lineage- and species-specific phenotypic traits. New genes created through retrotransposition are retrogenes that are devoid of introns. Furthermore, paralogues are subsequently created through segmental duplications and sequence modifications. Retrogenes could be re-activated by putative promoters and other transcription regulatory elements suitably located upstream of the retrogene insertion sites [1-3]. In the process of transcriptional re-activation, the newly arisen transcript may acquire one or more noncoding exons in the 5'-untranslated region (5'-UTR). In the context of our current knowledge of the generation of multiple transcripts from a single gene through alternative splicing [4,5], the term "retrogene" is used throughout this paper to mean the "genomic copy" of a gene that is consituted of a complete coding sequence without intron interruption taking no consideration on whether the resulting transcripts carry exonised sequences through alternative splicing. Whether or not "retrogene" should be redefined as such is debatable.

Many retrogenes are functional [6]. It has further been estimated that there are in excess of 1,000 transcribed retrocopies in the human genome over a tenth of which is biologically active [7]. Interestingly, the bulk of retrogenes is preferably expressed in the testis where the retrogene products actively participate in the spermatogenesis process and serve to further enhance biological functions unique to the male germline $[8,9]$. Transcription in the testis is not as tightly regulated as in other somatic tissues due to hyper transcription rates which could result in nondiscriminatory activation of otherwise imperfect or weak promoters [10]. Such a mode of promiscuous transcription, and possibly erratic alternative splicing processes, could lead to the generation of fortuitous testicular transcripts. Promiscuous transcription and transient transcriptional gene activation have also been shown to occur at the crucial stage of zygotic genome activation [11]. One outstanding feature of promiscuos transcription is excessive transcription of highly repetitive genomic sequences $[11,12]$. The bulk of genomic repetitive sequences are transposable elements (TEs). A significant number of mammalian genes has been shown to be regulated by transcriptional elements of the endogenous retroviruses (ERVs) or long-terminal repeats (LTRs) of TEs [13]. Evidence is emerging to suggest that ERVs and other TEs may constitute a critical driving force in speciation [14].

We have previously proposed the existence of a novel bipartite TDPOZ protein family members of which carry the TD (TRAF domain), also called MATH (meprin and TRAF homology, and POZ (poxvirus and zinc finger)/BTB (Broad complex, Tramtrack, Bric à brac) [15]. Almost all known eukaryotic TD proteins are known to be involved in the regulation of protein processing and ubiquitination [16]. The representative TD proteins, the tumour necrosis factor receptor-associated factors (TRAFs), bind to the tumour necrosis factor receptors or other adaptor molecules to participate in cellular proliferation and survival, and in cell-death signalling $[17,18]$. On the other hand, POZ proteins have been implicated in biological processes including DNA damage responses, cell cycle progression and in embryonic developmental events and hematopoietic stem cell fate determination [19]. The TD and POZ domains are found in separate proteins in association with other DNA-binding or protein-protein interacting domains except for the TDPOZ bipartite proteins that we have first reported $[15,20]$. Tdpoz genes are found in both higher and lower animals and in plants suggesting important biological functions. To date, the only functionally characterised mammalian TDPOZ protein is the nuclear speckle-associated protein SPOP. SPOP acts as an adaptor of Daxx in the ubiquitination process involving CUL3-based ubiquitin ligase contributing to regulation of Hedgehog/Gli signalling [21-24].

In the mouse and rat genomes, $T d p o z$ sequences appear in multiplicity as retrocopies of full-length or truncated coding sequences uninterrupted by introns $[15,20,25]$. Tdpoz retrogene multiplicity is found only in the two completely sequenced rodent genomes and not in other animals and plants suggesting that the Tdpoz retrogenes emerged after the evolutionary divergence of the rodent lineage. The first reported mouse Tdpoz retrogene, Tdpoz1 [GenBank:AF290198], is transcribed in the egg and in the preimplantation-stage embryo [15,20]. Other mouse Tdpoz retrogenes are subsequently identified and are found to be transcribed in low levels in the pre-implantation embryo and in the testis [15]. In the rat, the Rtdpoz-T1 and -T2 genes (abbreviated as $T 1$ and $T 2$ herein) [GenBank:AY902365 and AY902367, respectively] are transcribed specifically in the testis [20]. Database interrogations of the rat genome have further revealed 300 hits of Tdpoz homologous sequences, dubbed Rtdpoz for rat $T d p o z$, that show $>85 \%$ sequence identities with the $T 1$ or $T 2$ open reading frame (ORF); these sequence hits are distributed over seven different chromosomes of the rat genome. However, the bulk of the hits is found in a 2.5-Mb cluster in the Rn2_2148 supercontig [GenBank:NW 047626.2] mapping at 2q34 on chromosome 2 [20]. Active retrotransposition and duplication are thought to be the major forces driving the creation and expansion of the Rtdpoz sequence repertoire in the contemporary rat genome. In a $~ 800-k b$ region at the 2 q34 locus, twenty-six Rtdpoz retrogenes are discerned, including $T 1$ and $T 2$ [20]. The biological functions of the putative $\mathrm{T} 1$ and $\mathrm{T} 2$ proteins have yet to be elucidated. On alignment of the cDNA and genomic DNA sequences, it 
has been determined that the major 3'-terminal exon of the testicular $T 1$ and $T 2$ transcripts carry the uninterrupted coding sequences, qualifying $T 1$ and $T 2$ as retrogenes; however, the transcripts have also acquired two to three short noncoding exons in the $5^{\prime}$-untranslated region $\left(5^{\prime}\right.$ UTR) of the transcripts (Figure 1A). Intriguingly, all the T1 and $T 2$ transcripts share a common 5'-leader exon 1a sequence that appears only once at $2 \mathrm{q} 34$ and is also unique in the entire rat genome. How exon $1 \mathrm{a}$ is added to the 5 '-ends of both the $T 1$ and $T 2$ transcripts remains to be addressed. We have also previously reported a minor testicular transcript, dubbed T3, which appears to be a fusion of $T 1$ and $T 2$ exons [20]. Are there other T1-T2 "chimeric" transcripts, and how are these transcripts generated? In this work, we further explore other novel features in the T1 and $T 2$ transcripts in an attempt to further understand transcription and pre-mRNA processing of retrogenes.

\section{Results}

\section{Developmental regulation of TI and T2 expression}

To determine the expression status of the T1 and T2 genes during development, RNA was prepared from developmental stages between day 12 (E12) to day 20 (E20) just before birth. RT-PCR was performed using T1- or T2-discriminating primers located in different exon sequences of the genes (Figure 1A). The RT-PCR results show that $T 1$ was expressed only at stages E14 to E16 of development (Figure 1B, lanes 3-5) with a distinctive expression profile for each stage indicating differential $T 1$ transcription at these developmental stages. On the other hand, T2 was expressed up to E17 and the expression profile was rather consistent and was largely similar to that of the testis [20] except that at E16, extra bands were detected, and at E17 only the lower band was present (Figure 1B, lanes 9-14). The transcription profiling experiments, hence, establish that expression of the testicular $T 1$ and $T 2$ genes is developmentally regulated, and that there exists a notable disparity in the expression patterns of the genes at specific developmental stages. This suggests that transcription of the two genes, despite the sharing of the leader exon $1 \mathrm{a}$, is differentially regulated. The $T 1$ and $T 2$ genes, are silenced at day 17 (E17) and day 18 (E18) of development, respectively, when organogenesis is now completed and the foetus enters the active phase of growth and expansion.

\section{Assorted 5'-UTR structures derived from alternative splicing involving transposable element sequences}

To fully characterise the multiple $T 1$ and $T 2$ transcripts, a combination of RT-PCR and rapid amplification of cDNA ends (RACE) approaches was applied. In the RT-PCR approach, each of the RT-PCR bands was cloned and two to three clones generated from each RT-PCR product were randomly selected for sequence analysis (Figure 1B). In this effort, a total of eleven unique $T 1$ and $T 2$ transcript sequences were derived for all the expressing stages (Figure 2A and see below; designations of the RT-PCR-derived

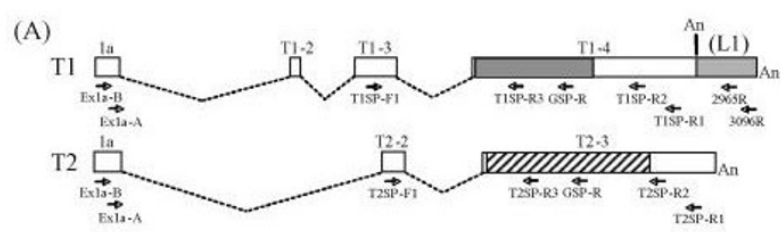

(B)

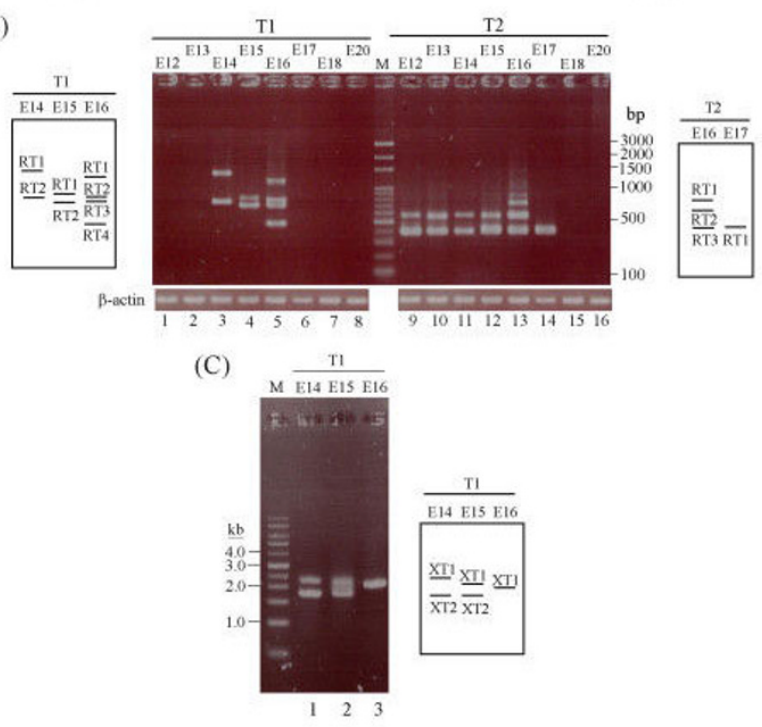

\section{Figure I}

Developmental regulation of Rtdpoz-TI and -T2. (A) Exons constituting the testicular $T I$ and $T 2$ transcripts. In both $T I$ and $T 2$, the common exon Ia is used. The noncoding sequences are shown as unfilled boxes. The uninterrupted $\mathrm{TI}$ - and $\mathrm{T2}$-coding sequences that reside in exons 4 ( $\mathrm{TI}-4)$ and $3(\mathrm{~T} 2-3)$ of the respective genes are shown as crosshatched or slanting-hatched boxes, respectively. In $T I$, the LI sequence (see text) in the 3'-UTR is shown in grey. The relative positions of the primers used in the RT-PCR expression profiling and the RACE analysis are shown (see Table 2 for primer sequences). An, polyA tract. (B) Developmental expression profiles of the $T I$ and $T 2$ genes. The developmental stages analysed were from day 12 (EI2) through to day 20 (E20). $\beta$-actin was included as a control. In the experiments, the ExIa-B + TISP-RI or ExIa-B + T2SP-RI primer pairs (see above) were used in the first-round PCR for $T I$ and $T 2$, respectively, followed by the use of the ExIa-A + TISP-R3 (for $T I$ ) or Exla-A + T2SP-R3 (for T2) primer pairs in the second-round PCR as detailed in the Methods section. On the left and right of the photo panels are schematic representations of the PCR bands with band designations as explained in the text. (C) 3'-Extended RT-PCR analysis of the TI transcripts in the developmental stages that expressed the gene. In the first-round PCR, the ExIa-B and 3096R primers (see (A) above) were used followed by a second-round PCR using the ExIa-A and 2965R primers as described in Methods. Band designations, prefixed by "XT", are depicted in the schematic drawing alongside the gel display.

transcripts carry the prefix "RT" in Figure 1B in the schematic drawings in the left and right panels). To define the 5'- and 3'-ends, RACE experiments were conducted. To 
(A) T1 transcripts

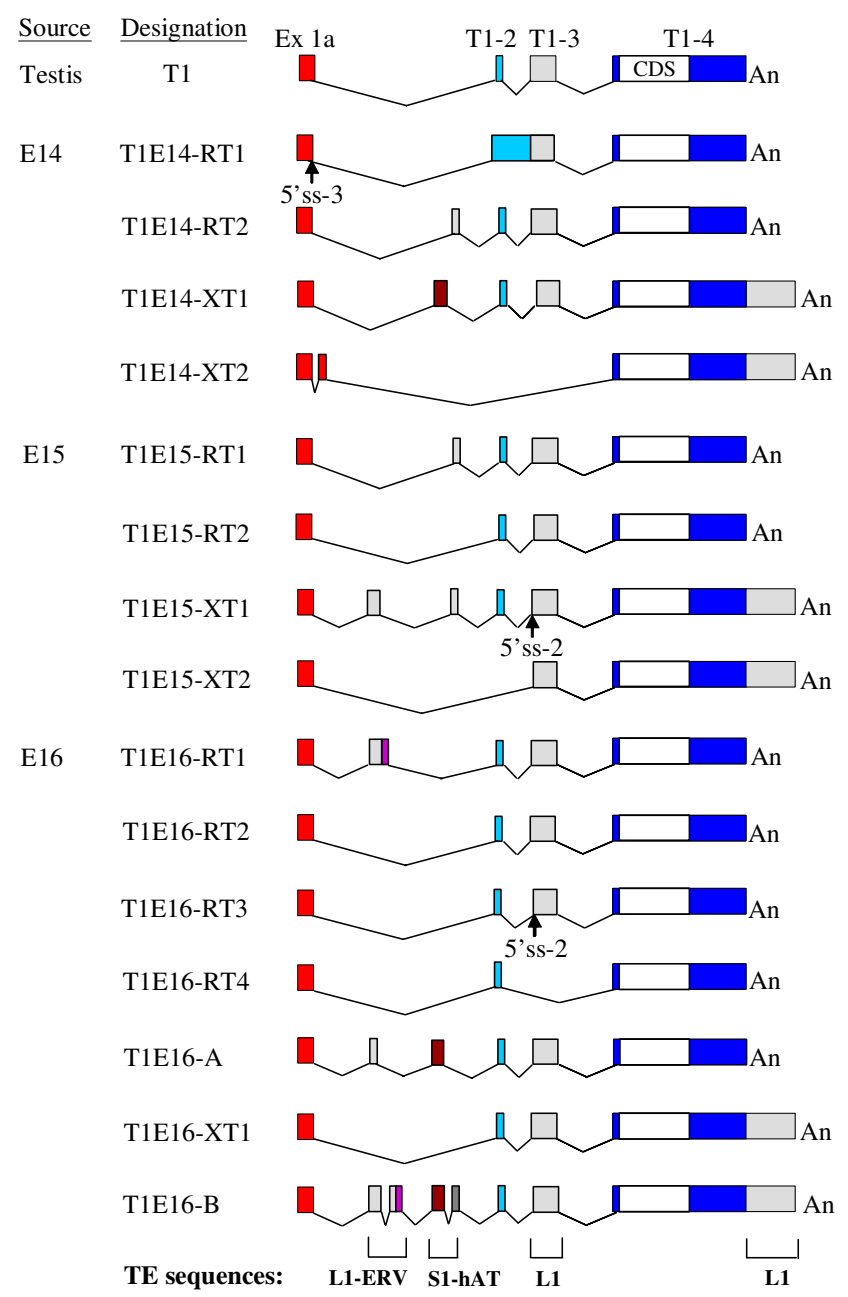

\section{(B) T2 transcripts}

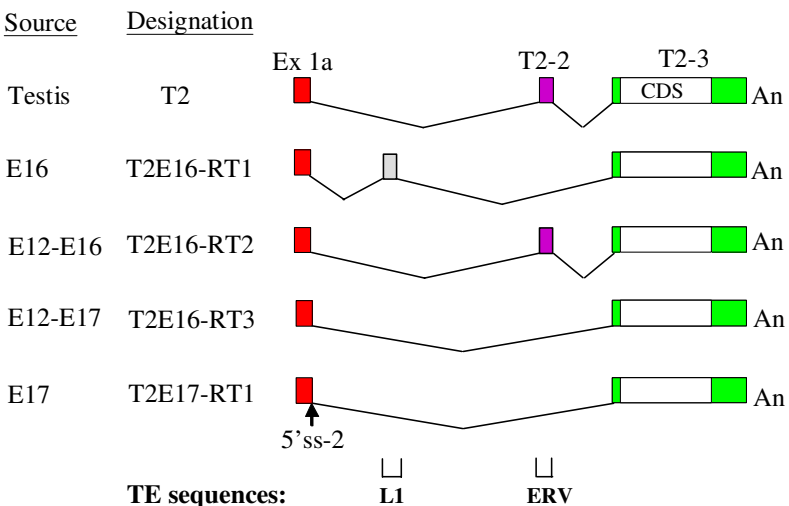

Figure 2

Exon map of the embryonic TI (A) and T2 (B) transcripts. The $T$ I and $T 2$ transcripts shown were derived by a combination of direct standard RT-PCR (transcripts designations with the "RT" prefix), 3'-extended RT-PCR (XT-tagged transcripts) and RACE experiments (transcripts TIEI6-A and -B) as described in the text. For identical TI or T2 transcripts derived from the various experimental approaches, only a representative transcript designation is used. Included as a reference is the testicular TI and T2 exon map that was previously derived [20]. Exons are represented by coloured blocks and the exon designations are shown above the testicular transcripts. The coding sequences (CDS) of $T I$ and $T 2$ are shown as white boxes. An, polyA tract. Alternative 5 '-splice sites (5'ss) are indicated. At the bottom of the $T I$ and $T 2$ transcript panels, the elucidated transposable element sequences are displayed aligning with the corresponding exon of the transcripts. LI, LINEI sequence; ERV, endogenous viral sequence; SI, SINEI sequence; hAT, DNA transposon. TIEI6-A and -B are now filed as GenBank:FJ004892 and GenBank:FJ004893, respectively. The scheme is not drawn to scale.

avoid discrimination between $T 1$ and $T 2$ in the RACE analysis and to increase specificity and sensitivity in detecting transcripts of low abundance, $T 1$ and $T 2$ consensus primers were used in the first-round cDNA synthesis followed by second-round nested PCR using T1- or T2specific primers. A total of forty-nine 5 '-RACE and twentyfive 3'-RACE clones were obtained and sequenced. The vast majority of the 5'RACE sequences were identical with the $T 1$ and $T 2$ transcript sequences obtained in the RTPCR experiments above. Moreover, all T1 and T2 transcripts carried the leader exon $1 \mathrm{a}$ and the 5 '-termini were largely similar to those first reported for the testicular transcripts [20]. In the 3'-RACE analysis, the sequences obtained for the $T 2$ transcripts at all developmental stages 
were identical to the 3 '-end of the testicular $T 2$ gene and were used to construct the full $T 2$ transcript sequences (Figure 2B). For the T1 transcripts, all except one 3'-RACE sequences were identical to the 3 '-end of the testicular T1 transcript. The novel T1 3'-terminal sequence (contained in the $T 1$ transcripts designated T1E16-B in Figure 2A) was a 542-bp 3'-extension of the regular 3 '-end for the $T 1$ transcripts; the extended 3' sequence also possesses a putative but non-canonical ATAAAA polyadenylation signal located 6-bp upstream of the polyA tail (see GenBank:FJ004893 for sequence details). A query of the GIRI RepBase database further revealed that the extension was a segment of the non-long terminal repeat (non-LTR) long interspersed element 1 (LINE1, or L1) sequence (Figure 2A). On the other hand, a similar but shorter transcript, T1E16-A [GenBank:FJ004892], lacks this L1 extension (Figure 2A).

The uncovering in the 3'-RACE experiments of the extended 3'-UTR sequence of T1 warranted further RTPCR analysis to confirm the authenticity of the 3'-extension and to further investigate if other $T 1$ transcripts carried similar 3'-extension. To achieve this goal, two-round nested RT-PCR was performed across the entire gene sequences stretching from the 5 '-terminal exon 1 a to the extended L1 sequence using primers Ex1a-B and 3096R followed by the use of primers Ex1a-A and 2965R (Figure 1A, T1 primer map). In the three T1-expressing developmental stages, one or two RT-PCR bands were discerned indicating, indeed, the existence of multiple $T 1$ transcripts with the 3'-extension (Figure 1C). The PCR products were subsequently cloned and sequenced and the sequences are designated with the prefix "XT" (Figure 2A). Fulllength XT transcripts are constructed assuming they share the same testicular 5'- and the 3'-end of T1E16-B (Figure 2A). A notable exception in the XT transcripts was T1E14XT2 which included a new 45-bp exon the sequence of which was located 887-bp downstream of the leader exon $1 \mathrm{a}$; this transcript also lacks the ubiquitous exons 2 and 3 (T1-2 and -3) of T1 (Figure 2A).

The sequences generated by RT-PCR and RACE were combined to construct full-length $T 1$ and $T 2$ transcript sequences as follows. For the T1 transcripts, 3'-RACE experiments generated two authentic 3'-ends: the first is as found in the testicular $T 1$ transcript and the second is represented by T1E16-B that carries an extended 3'-end resulting from incorporation of an L1 sequence. Hence, all "XT" transcripts (Figure 1C) were complemented with the extended 3'-end of T1E16-B whilst the remaining T1 transcripts were 3 '-tagged with the 3 '-terminal sequence of the testicular $T 1$ sequence (Figure 2A). For T2, all transcript sequences were tagged with the only 3 '-end of the testicular T2 derived by 3'-RACE (Figure 2B). To discern possible exon organisation, the transcript sequences were used to query the rat genome sequence (assembly version RGSC v3.4, as on December 1, 2008). All discernible exon sequences were qualified by the presence of the consensus splice junctions. Furthermore, all transcript sequences were also subjected to scrutiny for repetitive sequences by querying the GIRI RepBase database. It is noted in the RepBase analysis that the ubiquitous exon 3 of $T 1$ (T1-3) and exon 2 of T2 (T2-2) are derivatives of L1 and the endogenous viral (ERV) TE sequences, respectively. The derived exon organisation of the $T 1$ and $T 2$ transcripts is displayed in Figure 2. In the $T 1$ transcript category, transcripts identical to the testicular $T 1$ are detected at E15 (T1E15-RT2) and E16 (T1E16-RT2) but not at the E14stage of development. Most developmental transcripts retain either or both the testicular exons 2 and 3 (T1-2 and -3) except for T1E14-XT2 that has lost both exons. Furthermore, T1E14-RT1 has retained the 534-bp intron separating exons 2 and 3. More significantly, seven T1 transcripts carry superfluous exons sandwiched between exons $1 \mathrm{a}$ and 2 (T1-2) (Figure 2A). The T1E16-B transcript appears to embody all these superfluous exons in two clusters derived from two distinct genomic sequences, designated as L1-ERV and S1-hAT for convenience of description, whereas other transcripts carry alternatively spliced segments of these two genomic sequences. The L1ERV sequence was a composition of a $\sim 170$-bp relic sequence of the highly repetitive $\mathrm{L} 1$ fused to a short remnant of ERV sequence (Figure 3A; see also Additional file 1 , panel A for sequence details). The S1-hAT genomic segment is a 1 .9-kb sequence composed of eight short interspersed element 1 (SINE1) remnant sequences and the hAT (for hobo from Drosophila, Ac from maize, and Tam3 from snapdragon) DNA transposon [26,27] (Figure 3B; also Additional file 1, panel B). In a BLAST query, the S1hAT sequence was further found to be duplicated in tandem in chromosome 2 and an almost identical copy was also discerned in chromosome 3 of the rat genome (Figure $3 \mathrm{~B})$. Different spliced segments of these two genomic sequences are stitched to different $T 1$ transcripts using the splice sites depicted in Figure 3. For example, the allencompassing T1E16-B transcript harbours two L1 and two ERV subfragments of these two TE genomic sequences (Figures 2A and 3). Incorporation of extraneous TE-associated exons results in extended 5'-UTRs in the T1 transcripts with potential biological consequences as is demonstrated below.

On the other hand, the developmental $T 2$ transcripts are uncomplicated in exon organisation (Figure 2B). All developmental $T 2$ transcripts also carry the leader exon 1a and the uninterrupted coding exon 3 (exon T2-3). The testicular exon 2 (T2-2), which itself is an ERV remnant, may or may not be associated with the developmental $T 2$ transcripts. In T2E16-RT1, a superfluous exon derived from L1 is found located between the constitutive exon 1a and the 
(A) The L1-ERV sequence

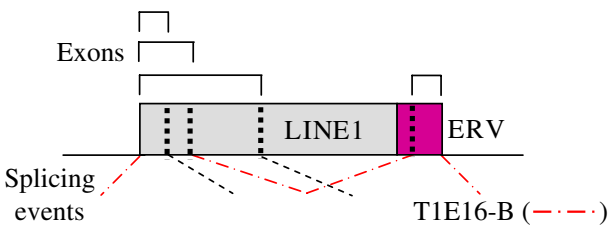

(B) The S1-hAT sequence
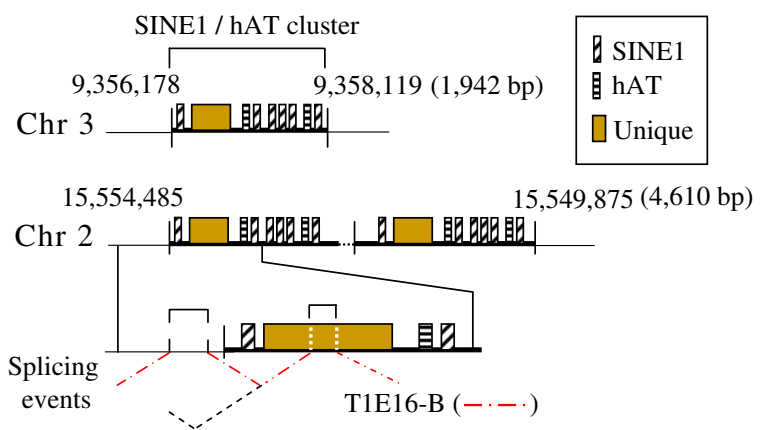

\section{Figure 3}

Alternative splicing of two genomic regions of transposable elements with contribution to $T I$ transcripts. The genomic regions are as defined in Figure 2A and Table I. Sequence details are found in Additional file I. (A) The LIERV segment is a composite of LINEI (LI) (in grey) and ERV (in purple) sequences. The roofs above the TE sequences represent exons resulting from alternative splicing as demarcated by vertical dotted lines in the boxes representing TEs. Slanting dashed lines in black denote splicing events that generate the discerned exons. The dashed-dotted lines in red indicate two consecutive splicing events in this region that have led to the two TE-associated exons in the TIEI6-B transcript. (B) The SINEI (SI)-hAT sequence as defined in the text. There are two tandem copies of SI-hAT on chromosome 2 (in the Rn2_2I 48 supercontig [Gen-

Bank:NW_047626.2], RGSC ver3.4) and a single copy on chromosome 3 (Rn3_2179 supercontig, [Gen-

Bank:NW 047657.2]) of the rat genome; the nucleotide positions and the sizes of the respective sequence copies in these chromosomal segments are indicated. At the bottom, splicing events and the resulting exons are depicted using the same symbols as for (A) above. The scheme is not drawn to scale.

coding exon 3 replacing exon T2-2 (Figure 2B). The T2 transcripts may be simpler in exon organisation but they are still tinted by TE-derived exons.

In summary, sequence analysis of the $T 1$ and $T 2$ transcripts indicates extensive alternative splicing events involving sequences of various highly repetitive TE sequences contributing to the 5'-UTR of the transcripts, particularly the $T 1$ transcripts. In the 3'-UTR, two major termination sites were elucidated for the $T 1$ transcripts with the 3'-distal termination site contributed by L1. It is also observed that the overall exon organisation of the T2 transcripts is uncomplicated whereas the $T 1$ transcripts vary extensively in the 5'-UTR structure when expressed during development.

\section{Detection of TI-T2 chimeric transcripts}

In our previous work, we presented a testicular transcript which was a composite of $T 1$ and $T 2$ sequences [20]. To authenticate T1-T2 chimerism in this work, RT-PCR was performed using a T1- or T2-exon 2-specific forward primer in mix-gene combinations with a $T 2$ or $T 1$ reverse primers located at the 3'-UTR of the respective gene for detection of possible T1-T2 or T2-T1 exon constitutions. To increase sensitivity and specificity, two rounds of nested PCR were performed: the first-round PCR was done using an exon 1a primer (Ex1a-B in Figure 1A) common to both $T 1$ and $T 2$, and a $T 1$ or T2 3'-UTR sequencespecific reverse primer (T1SP-R1 or T1SP-R2 in Figure 1A). In the PCR, plasmids carrying the $T 1$ or $T 2$ cDNA sequence were included as controls; these plasmids generated positive bands corresponding to the respective gene indicating gene specificity (Figure 4A, lanes 1 and 2). When the testicular mRNA was used in the first-round RTPCR using the T1 or T2 primers, two major bands were discerned (Figure 4A, lane 3). For subsequent PCR, T1- or T2-specific exon 2 forward primer (T2SP-F1 or T1SP-F1) was used in a mix-gene fashion in combination with the T2- or T1-specific reverse primer (T1SP-R1 or T2SP-R2) located at the 3'-UTR sequence of the respective gene (Figure $4 \mathrm{~B}$; see also Figure $1 \mathrm{~A}$ for primer map positions). In the control experiments in which the T1 and T2 plasmids were also tested in the mix-gene reactions, no PCR products were detected as expected for such mono-gene scenarios (Figure 4A, lanes 4 and 5). However, when the testicular first-round cDNA products were subjected to the mix-gene PCR, two distinct bands were now discerned in each of the mix-gene reactions (Figure 4A, lane 6).

The PCR bands were excised and cloned; two or more clones derived from each of these bands were sequenced; the sequences are given the prefixes $\mathrm{T} 21$ or T12 in the order of appearance of the $T 1$ and $T 2$ sequences. Exon mapping of the sequences derived clearly shows that the mix-gene PCR products are T2-T1 or T1-T2 chimeric transcripts defined by accurate splice-site demarcation (Figure $4 \mathrm{~B})$. The $1.7-\mathrm{kb}$ T21Te-RT1 sequence is composed of the T2 exon 2 and partial exon 3 truncated within the coding sequence, and the T2 exons are accurately spliced to the T1 exons 2, 3 and the full coding sequence of exon 4 . The 1.4kb T21Te-RT2 sequence carries T2 exon 2 (T2-2) which is spliced to exons 2, 3 and 4 of $T 1$ (T1-2, -3, and -4). Conversely, the $1.2-\mathrm{kb}$ T12Te-RT1 sequence is composed of the $T 1$ exon $2(\mathrm{~T} 1-2)$ spliced to exon 2 and the coding exon 3 of T2 (T2-3 and -3). The T12Te-RT2 sequence was 
(A)

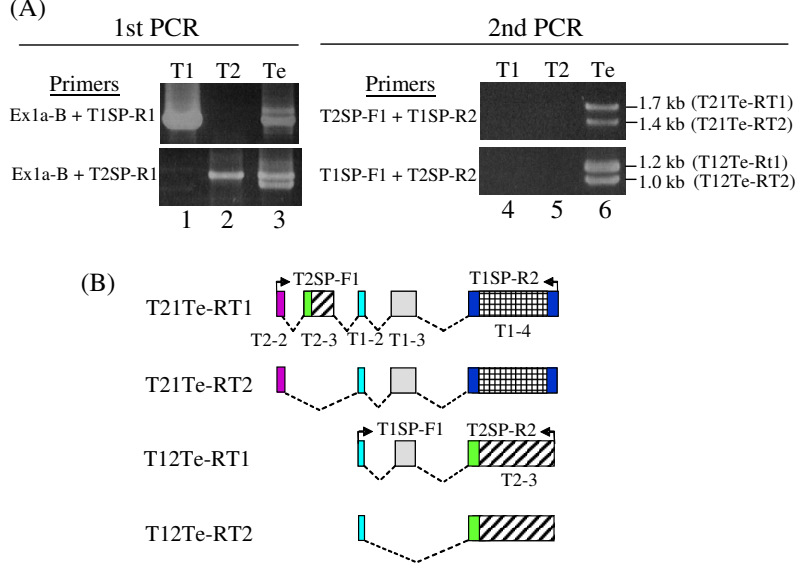

Figure 4

Detection of TI-T2 chimeric transcripts in the testis. (A) RT-PCR profiling of $T I-T 2$ chimeric transcripts. Two rounds of nested PCR were performed using oligo(dT)primed RT products of the testis mRNA. In the first-round PCR, the consensus ExIa-B and the TI- or T2-specific 3'UTR primers, TISP-RI and T2SP-RI, respectively, were used (see Figure IA for relative positions and Table 2 for sequence details); in the second-round PCR, the exon $3(T I)$ or exon 2 (T2)-based T2SP-FI or TISP-FI primers were used in combination with TISP-RI and T2SP-RI. The TI and $T 2$ plasmids were used as controls in both rounds of PCR (lanes I-2 and 4-5). The PCR products (with designations in brackets) generated in the second-round PCR (lane 6) were cloned and sequenced. (B) Schematic depiction of the PCR products derived from (A). The exons of the transcripts are shown using the same colour code and exon designation as in Figure 2. Primers used in the second-round PCR are also shown.

simplest in structure in being composed of $T 1$-exon 2 coupling with the coding T2-exon 3. Since these transcript sequences were RT-PCR products obtained rather forcefully through two rounds of PCR, they most likely represent minor populations of authentic T1-T2 chimeric transcripts. The naturally occurring 5'- and 3'-ends had not been determined for these chimeric transcripts. We have, thus, produced further experimental evidence that T1-T2 or T2-T1 chimeric transcripts do exist and their existence raises the question on how these transcripts are generated

\section{Embedment of TI and T2 exons in a minefield of TE sequences}

Exon mapping reveals that the $T 1$ and $T 2$ exon sequences are dispersed over $\mathrm{a} \sim 700 \mathrm{~kb}$ segment in the sequence of the rat Rn2_2148 supercontig [GenBank:NW 047626.2]. Using a threshold of $>98.5 \%$ sequence identity in BLASTbased queries, the relative physical locations of all the T1 and $T 2$ exons are derived as shown in a linear order in

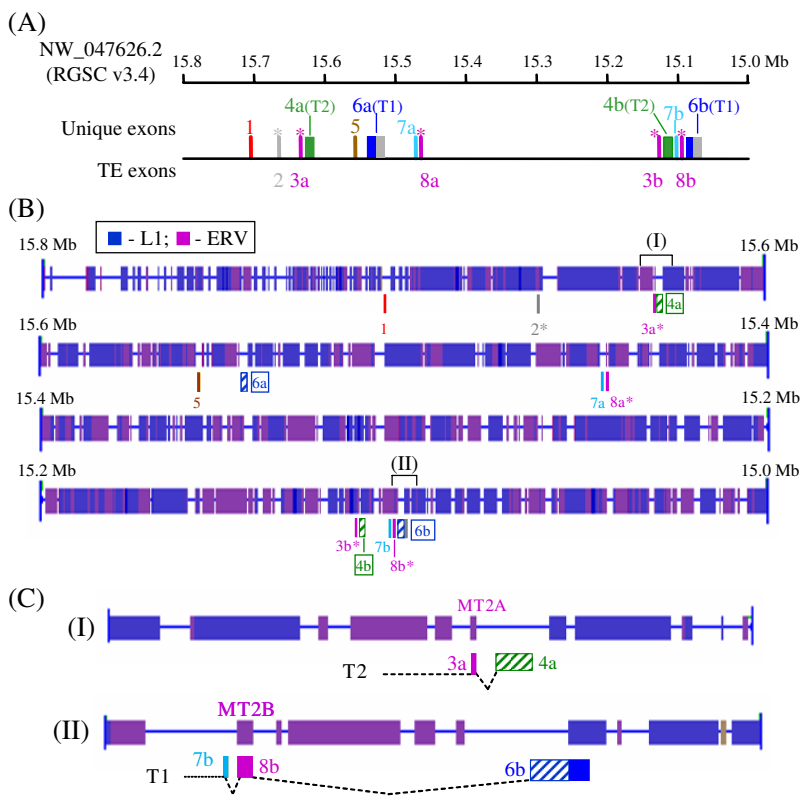

\section{Figure 5}

Exon assemblage and exonisation of TE sequences into the 5'-UTRs of the TI and T2 transcripts. (A) A chromosomal map of discerned exons of TI and T2 transcripts derived by bioinformatics-based alignment of the transcript and the genomic sequences. The relative map positions of the exons are in a linear order in the defined regional sequence of the Rn2_2I48 supercontig. The new exon designations used are as defined in Table I. Exons that are derived from TE sequences are asterisked with the exon designations shown below the exon map; exons of unique sequences are shown above the map. The $T I$ and $T 2$ coding exons are shown in parenthesis. The scheme is prepared only to an approximate scale. (B) Embedment of $\mathrm{TI}$ and $\mathrm{T2}$ exons in a dense field of TE sequences. The nt 15,800,000-15,000,000 (I5.8 Mb-I5.0 Mb) chromosomal segment of the Rn2_2I48 supercontig that harbours all the discerned $T I$ and $T 2$ exons, the LI (shown in blue) and the ERV sequences (in purple) are shown. The exons (see Table I) are mapped below the TE sequences using the same colour code as in Figures 2 and 5A above. Coding exons are denoted by hatched bars and the boxed-in exon designations. Roofed segments (I) and (II) are shown in further details in (C). (C) Expanded views of the roofed segments of the $T 2$ and $T /$ aligning exons displayed in (B) above. The respective TE and coding sequences are shown.

Table 1 and Figure 5A. An important outcome of this analysis is the finding of duplicated cassettes of the T2 exons 2 and 3 (T2-2 and T2-3) each with a short intron of $\sim 500$ bp separating the exons (Table 1, solid boxes). For T1, there are also two cassettes of exons 2, 3 and 4 (T1-2, -3 and -4) (Table 1, dashed boxes). T1 exons 2-4 in the second (downstream) cassette are arranged in a linear order and are separated by relatively short introns. However, the 
Table I: Rtdpoz-TI and -T2 exon assembly

\begin{tabular}{|c|c|c|c|c|c|}
\hline Exon & & Size (bp) & Rn_2I48 position (nt) & Intron $(\mathrm{kb})$ & Exon description \\
\hline Previous & New & & & & \\
\hline $\mathrm{Ia}$ & 1 & 169 & $|5,707,883-| 5,707,8 \mid 4$ & 39.40 & Major exon I for $T I$ and $T 2$ \\
\hline LI-ERV & 2 & & $15,668,417-15,666,808$ & 31.15 & nonLTR-LI \& ERV TE sequences \\
\hline T2-2 & $3 a$ & 157 & $15,635,659-15,635,503$ & 0.563 & ERV-MT2A TE sequence \\
\hline *T2-3 & $* 4 \mathbf{a}$ & $\mathbf{I}, \mathbf{5 5 0}$ & $|5,634,940-| 5,633,39 \mid$ & 79.60 & T2 coding sequence \\
\hline SIhAT & 5 & $279 / 36$ & $15,553,789-15,550,897$ & 9.98 & Unique sequences amidst SINE/hAT \\
\hline \multirow[t]{2}{*}{$* \mathbf{T I}-4$} & $* \mathbf{6} \mathbf{a}$ & $\mathbf{1 , 5 3 0}$ & $|5,540,9| 5$ - | 5,539,386 & 64.6 & TI coding sequence \\
\hline & & & $(15,538,842)$ & & (3' LI extension) \\
\hline $\mathrm{TI}-2$ & $7 a$ & 73 & $15,474,800-15,474,727$ & 0.534 & Unique sequence \\
\hline $\mathrm{TI}-3$ & $8 a$ & 292 & $15,474,193-15,473,900$ & 358.1 & ERV-MT2B TE sequence \\
\hline
\end{tabular}

\begin{tabular}{|c|c|c|c|c|c|}
\hline T2-2 & $3 b$ & 157 & $15,115,847-15,115,693$ & 0.470 & ERV-MT2A TE sequence \\
\hline *T2-3 & $* 4 \mathrm{~b}$ & I,550 & $|5$,$| I 5,223 - I 5, I | 3,674$ & 12.45 & T2 coding sequence \\
\hline $\mathrm{TI}-2$ & $7 b$ & 73 & $15,101,224-15,101,15 \mid$ & 0.536 & Unique \\
\hline $\mathrm{TI}-3$ & $8 b$ & 292 & $15,100,615-15,100,323$ & 4.93 & ERV-MT2B TE sequence \\
\hline *T I-4 & $* 6 \mathbf{b}$ & 2,075 & $\begin{array}{c}15,095,393-\mid 5,093,863 \\
(15,093,32 \mid)\end{array}$ & -- & $\begin{array}{l}\text { TI coding sequence } \\
\text { (3' LI extension) }\end{array}$ \\
\hline
\end{tabular}

Sequences with $>98.5 \%$ identity between the $T I$ and $T 2$ transcript sequences and the genomic sequence of the Rn2_2I 48 supercontig (NW_047626.2, RGSC v3.4) are listed. Exons are tabulated in order of appearance in the genomic sequences. Putative TI and T2 coding exons are shown in heavy letters and are asterisked. The solid and dashed boxes denote the unique T2- and TI-specific exons, respectively, that are duplicated in different chromosomal locations. The relative exon positions are schematized in Fig. 5A.

exons in the first (upstream) cassette are arranged disorderly in a 4-2-3 exon configuration, and the coding exon 4 is mapped $64.6 \mathrm{~kb}$ upstream of the tight exons $2-3$ doublet. A comprehensive exon map is constructed to include all the identified exons; the exons are also re-named based on the linearity of appearance and their presence in the $T 1$ and $T 2$ transcripts (Table 1 and Figure 5A). In the revised exon designation scheme, the numerical indicates the order of appearance of the exons; suffices "a" and "b" are used to denote different alleles of the same exons.

As presented in the preceding sections, numerous $T 1$ and $T 2$ exons are derivatives of the highly repetitive sequences of the L1 and ERV transposable elements (Table 1 and Figures 2 and 3 ). When the $\sim 700-\mathrm{kb}$ genomic sequence that harbours all the $T 1$ and $T 2$ exons were subjected to a RepBase query, the sequence was found to be heavily mined with relics of TE sequences. An average TE occupancy of $\sim 60 \%$ is computed; some segments contain as high as $\sim 70 \%$ TE sequences (Figure 5B). In this genomic region, the unique $T 1$ and $T 2$ exons are precariously embedded within the TE minefield. When a transcription readthrough primary transcript carries such a heavy loading of redundant TE sequences, TE sequences that have devel- oped favourable splice junctions could readily be harvested as exons and be inducted into mature transcripts as typically exemplified by the $T 1$ transcripts T1E16-A and T1E16-B (Figures 2 and 3). In the more simplistic T2 splicing, the ERV-MT2A has become an almost permanent landmark of the T2 transcripts harvested as exon 3a (T22) (Figure 5C, panel I). In the same token, the ERV-MT2Bderived exon $8 \mathrm{a}$ or $8 \mathrm{~b}$ (T1-3) has also become a permanent fixture of the $T 1$ transcripts (Figure $5 \mathrm{C}$, panel II). At the 3 '-end, transcription read-though of the regular transcription termination site into a downstream L1 sequence has also resulted in an extended 3'-UTR in a significant population of $T 1$ transcripts (Figure $2 \mathrm{~A}$ ).

\section{A model of T2 and TI transcription and post- transcriptional processing}

Only one copy of exon 1 (previously called exon 1a) could be identified in the $700-\mathrm{kb}$ genomic sequence and also in the entire rat genome. When the completed mouse genome was interrogated for possible presence of the rat exon 1-like sequence, only one exon 1 copy was found on chromosome 3 [GenBank:NT_039240.7] with a 75.1\% sequence identity but there were short $\sim 50$-bp segments showing $>90 \%$ identities between the two sequences (see 
Additional File 2). When longer (1.5 kb) genomic sequences encompassing exon 1 and about 1-kb upstream sequences of the two rodents were aligned, sequence identity remained high at $69.4 \%$ (data not shown) indicating evolutionary relatedness. The described mouse-rat genomic sequence identity further supports the uniqueness of the exon 1 sequence in the rat genome. Interestingly, this exon 1 sequence is found only in the rodent genomes and not in the genomes of other animals and plants examined (data not shown) suggesting that the exon had evolved independently since the branching out of the rodent lineage.

Since all $T 1$ and $T 2$ transcripts carry the unique exon 1, our results clearly suggest that both genes are transcribed using a common promoter associated with this leader exon. Based on the derived exon order depicted in Figure $5 \mathrm{~A}$, the generation of the $T 2$ transcripts is a simple and direct affair through cis splicing of the proposed Pri-A primary transcript (Figure 6, panel A). (Note that in Figure 6, the splicing of only representative transcripts is shown.) To explain the generation of the $T 1$ transcripts initiating from the exon 1 promoter, transcription read-through of the $T 2$ exon cluster and a run up to the 3 '-distal exon cluster would have to be invoked to first generate a 600-kb Pri-B1 primary transcript that terminates at the T1-coding exon 6b (or T1-4 in the previous terminology) followed by appropriate cis splicing (Figure 6, panel B). The downstream $T 1$-coding exon $6 \mathrm{~b}$, but not the upstream exon $6 \mathrm{a}$, would have been used due to the fact that the other preceding exons, exons $7 \mathrm{a}$ and $8 \mathrm{a}(\mathrm{T} 1-2$ and -3$)$, are in the correct linear order in the downstream cluster but not the upstream exon cluster (exons 6b-7a-8b). In this scheme, it could not, however, be ascertained if the upstream exons $7 \mathrm{a}-8 \mathrm{a}$ or the downstream $7 \mathrm{~b}-8 \mathrm{~b}$ exon doublet is used. To explain the generation of the T1-T2 chimeric transcripts, transcription initiating from the exon 1 promoter and the generation of the transcription read-through primary transcripts Pri-B1 and -B2 would have to occur followed by alternative splicing (Figure 6, panel C). For example, the T12Te-RT1 chimera would have used the upstream exon 7 a (T1-2) that normally appears in $T 1$ transcripts splicing to the downstream $T 2$ noncoding exon $3 \mathrm{~b}(\mathrm{~T} 2-2)$ and the coding exon 4b (T2-3); the T21Te-RT1 chimera would have used the T2 exon $3 \mathrm{~b}$ (T2-2) stitching to the T1 exons $7 \mathrm{~b}-8 \mathrm{~b}-6 \mathrm{~b}$ cluster.

Taken together, the proposed exon organisation offers a model of co-transcription and post-transcriptional processing to explain the structure of the $T 1$ and $T 2$ transcripts. Firstly, the close proximity of the leader exon 1 with the $T 2$ exons (exons 1, 3a and 4a) explains why the $T 2$ transcripts are uncomplicated with fewer splice variants whereas the $T 1$ transcripts are highly erratic and are frequently infused with TE sequences due to the extended size of the proposed primary transcripts. Secondly, the
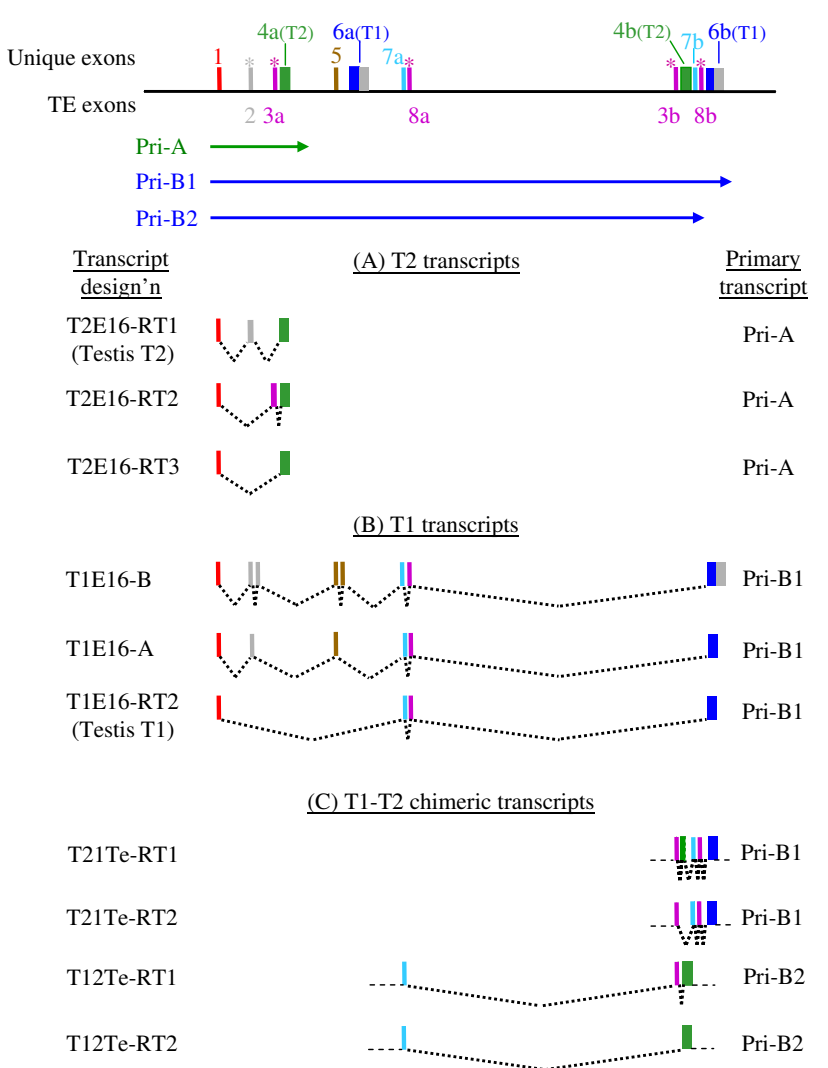

\section{Figure 6}

A model of co-transcription, alternative splicing and exonization of TE sequences in the generation of the TI (A), T2 (B) and TI-T2 chimeric (C) transcripts.

The ordered exon assemblage shown at the top of the scheme is as depicted in Figure 5A. Primary transcript A (Pri$A$ ) is proposed for the generation of the $T 2$ transcripts, and the transcription read-through primary transcripts Pri-BI and $-B 2$ are proposed for the generation of the $T I$ and $T I-T 2$ chimeric transcripts, respectively, as explained in the text. In the figure, only transcripts that exemplify representative exon usages are shown. Exons $4 a$ and $4 b$ and exons $6 a$ and $6 b$ carry the $T 2$ and $T I$ coding sequences, respectively, as indicated. Since chimeric sequences in panel (C) were derived by RT-PCR, only the derived exon sequences are shown. See also Figure 5 legend for an explanation of the symbols and colour code used.

model also dissembles that chimeric transcripts in both the $T 1-T 2$ and $T 2-T 1$ orientations are generated as rogue transcripts that have acquired illegitimate exons of the cousin gene through erratic alternative splicing. Although there is no evidence of alternative trans splicing, this possibility could not, however, be completely ruled out.

\section{Translational repression by TE-derived uAUGs and uORFs of TI transcripts}

In the 5'-UTR sequence of the testicular $T 1$ transcript, we could discern three upstream AUGs (uAUGs) and two upstream open reading frames (uORFs) of 21- and 75-bp 
in length both of which are derived from the inserted TE sequence (exon $8 b$, or T1-3); the upstream 21-bp uORF is in the same reading frame with the $T 1$ coding sequence (Figure 7A). The 5'-UTR-truncated T1E16-RT4 transcript lacks the TE insertions and, hence, the two uORFs in the 5'-UTR (Figure 7A). In the lengthy 5'-UTR of the T1E16-A transcript the bulk of which is composed of ERV and L1 sequences (Figure 2A), a total of twelve uAUGs and seven $\mathrm{uORFs}$ are discernible in all three reading frames with sizes ranging from $21 \mathrm{bp}$ to $123 \mathrm{bp}$ (Figure 7A).

To investigate if TE-containing 5'-UTR sequences of different $T 1$ transcripts contribute to regulation of gene expression, the 5'-UTR sequences of the T1, T1E16-RT4 and T1E16-A transcripts were inserted before the luciferase gene under the regulation of the SV40 promoter and an SV40 enhancer; the constructs were used in transient transfection of the Chinese hamster ovary cell line, CHO$\mathrm{K} 1$, followed by luciferase activity assays (Figure 7B). The results show that the luciferase activities under the regulation of the UAUG- and uORF-free T1E16-RT4 were maximal in the presence or absent of the SV40 enhancer. On the other hand, the uORF-abundant 5'-UTR of T1E16-A resulted in the lowest levels of luciferase activity whereas the T1 construct with two uORFs showed intermediate level of luciferase activity. Similar albeit lower relative luciferase activities were obtained using the testicular cancer cell line LC-540 in similar transfection and luciferase assays (data not shown).

To determine if the varied luciferase activities observed are associated with differential RNA stabilities, total RNA was prepared from the transfected cells at different post-transfection time points for RT-PCR analysis. At each of the time points examined, the luciferase mRNA level was found to be comparable for the three 5'-UTR constructs indicating similar luciferase mRNA stability despite the presence of different 5'-UTR sequences (Figure 7C). To discern possible regulation at the translation step, western blot analysis of lysate of the same sets of transfected cells was performed using an anti-luciferase antibody. The results show that the luciferase protein level was maximal for T1E16-RT4, minimal for T1E16-A and intermediate for $T 1$, in direct agreement with the relative luciferase activities determined above (Figure 7D). The effects of uAUGs and uORFs on translation were further supported by data derived from mutagenesis analysis of the two uORFs of $T 1$ in the luciferase constructs (Figure 7E). On removal of either or both the UORFs of $T 1$, luciferase activities were partially restored. Our data collectively indicate that different 5 '-UTR sequences in the $T 1$ transcript variants carrying different numbers of TE-derived uAUGs and uORFs could result in repressed translation of the $T 1$ gene.

\section{Discussion}

In a previous work, we first described testis-specific transcription of the Rtdpoz-T1 and -T2 genes [20]. In this work, we show that $T 1$ and $T 2$ are also transcribed in the developing embryo (Figure 1). More significantly, we show that each of the uninterrupted $T 1$ and $T 2$ coding exons are duplicated and the exons are embedded in a dense field of TE sequences. Consequently, the embryonic T1/T2 transcription displays two novel features: co-transcription of the two genes and frequent exonisation of TE sequences into the 5'-UTRs of the transcripts.

\section{Developmentally-regulated T2 and TI co-transcription}

Co-transcription of $T 2$ and $T 1$, in this gene order, is proposed based on the observation that all testicular and developmental $T 2$ and $T 1$ transcripts discerned share a unique exon 1 that resides upstream of the T2/T1 exon assemblage (Figures 2 and $5 \mathrm{~A}$ ). The exon 1 sequence is also found to be conserved as a unique sequence in the mouse genome (see Additional file 2) but not in the genomes of other animals and plants examined, consistent with evolutionary relatedness between the mouse and rat genomes. T2-T1 co-transcription implies that the genes are co-ordinately controlled by a common promoter and associated regulatory sequences.

T2 transcription is found to occur throughout the E12 to E17 developmental stages analysed and is silenced beyond E17. On the other hand, $T 1$ transcription is restricted only to E14-E16. In rodents, the organogenesis phase of embryonic development comes to an end at about E14-E15 from which point on active foetal growth occurs, a process that involves active cellular proliferation as opposed to active differentiation during the organogenesis phase [28]. Our data collectively suggest that $T 2$ expression is a normal monogenic transcriptional event that uses the AAUAAA polyadenylation signal located 10 nucleotides upstream of the polyA tract of the mature $T 2$ transcripts [GenBank:AY902367 and ref. [20]] and. On the other hand, $T 1$ expression is only realised when T2-to$T 1$ transcription read-through occurs to transcribe the $T 1$ coding exons (Figure 6). The occurrence of T2-to-T1 cotranscription may be attributed to high rates of transcription associated with active foetal growth similar to hyper transcription rates that have been shown in the testis [10]. The silencing of T1/T2 transcription at E17-E18 does not seem to involve hypermethylation of the exon 1 promoter which is unmethylated, despite the presence of a $\mathrm{CpG}$ island, in the testis and in all the developmental stages examined irrespective of $T 1 / T 2$ expression (unpublished data). It remains to be investigated if developmental and testicular transcription of the T2/T1 locus involves chromatin remodelling or the availability of positive or negative trans-acting factors. 
(A)

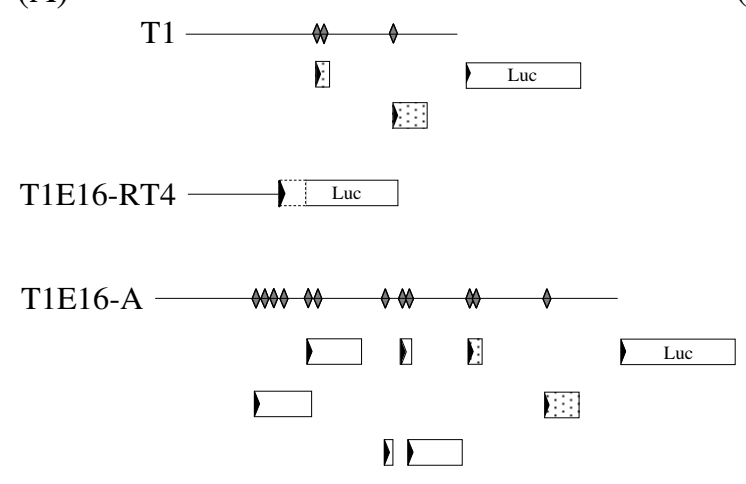

(C)

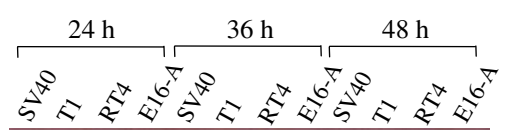

Luc

$\beta_{\text {-actin }}---------\cdots$
(B)
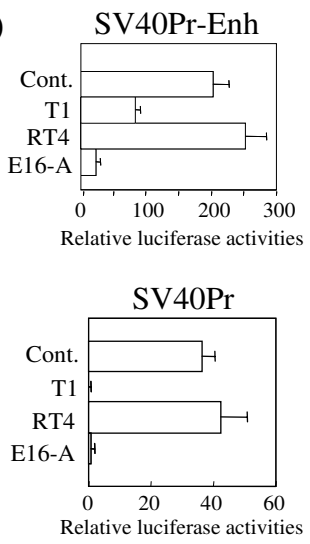

(D)

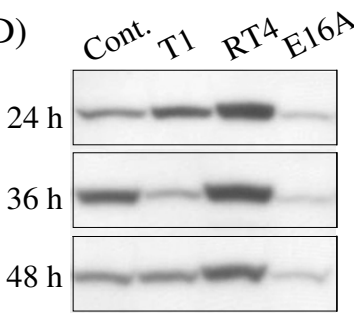

(E)
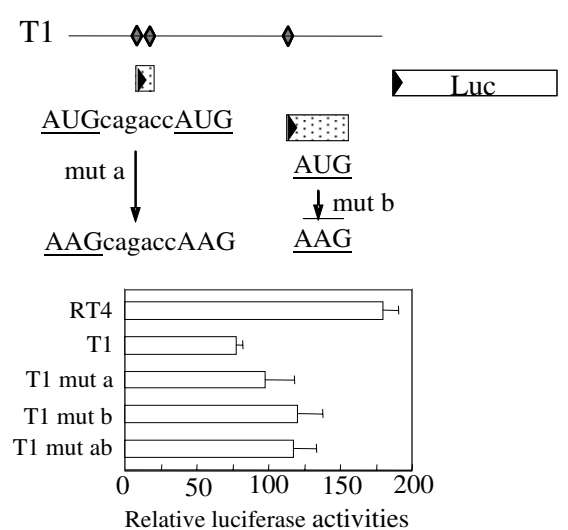

\section{Figure 7}

Translational repression by uORFs of selected TI transcripts. (A) Identification of UAUG (depicted by diamonds) and uORFs (boxes, with the vertically positioned triangles indicating AUG) in the 5'-UTR sequences (horizontal lines) of selected TI transcripts. Dotted boxes are uORFs found in the testicular TI transcript. The depicted 5'-UTR sequences were placed before the luciferase (Luc) gene in the pGL3-Control (SV40Pr-Enh) or pGL3-Promoter (SV40Pr) vectors. In the TIEI6-RT4 construct, a new initiation codon was fortuitously created by the cloning process adding 16 amino acid residues (dashed box) to the luciferase protein which did not affect the luciferase activity. (B) Transient transfection of CHO-KI cells and luciferase assays using the constructs described in (A). Transfection with the pGL3-Control or pGL2-Promoter plasmid was used as a positive control (Cont.). The data shown are from three independent experiments. (C) RT-PCR analysis of relative luciferase mRNA levels in the transfected CHO-KI cells. Transfected cells were harvested at the indicated time points for total RNA preparation and semi-quantitative RT-PCR analysis using luciferase gene-specific primers (Table 2). (D) Western blot analysis of the luciferase protein levels in the transfected CHO-KI cells at different post-transfection time points using an anti-luciferase antibody. The control (Cont.) data were derived from mock transfection using the blank vector. (E) Effects of disruption of uORFs on expression of the luciferase reporter gene. The three uAUGs and the two uORFs of the TI transcript, shown in (A) above, were disrupted by site-specific mutagenesis at the initiation codons as shown: mut a was a double mutant of the two uAUGs (underlined) found in the first uORF; mut b removed the uAUG in the downstream uORF whereas mut ab was a mutant in both the up- and downstream uORFs. The mutagenised constructs were used in transient transfection of the $\mathrm{CHO}-\mathrm{KI}$ cells followed by luciferase assays. The data shown in the bottom panel were derived from three independent transfection experiments. The parental TI and the uORF-free TIEI6-RT4 were included for comparison. 


\section{Exonisation of TE sequences into 5'-UTRs of the TI and T2 transcripts}

The most notable finding of this work is the high rate of exonisation of TE sequences into the 5'-UTRs of the T1 and $T 2$ transcripts through alternative splicing. In some T1 transcripts, an alternative transcription termination site is found in an L1 sequence located downstream of the constitutive site (Figure 2A). Frequent TE exonisation is clearly associated with the embedment of the constitutive exon 1 and the uninterrupted $T 1$ and $T 2$ coding exons in a $\sim 700-\mathrm{kb}$ chromosomal segment that is heavily populated with TE sequences (Figure 5). In this segment, the computed average TE content is $60.7 \%$, much higher than the mean TE content of $40 \%$ in the rat genome $[29,30]$. Notably, the second exon of the T2 gene (T2-2 or exon 3a/ $3 \mathrm{~b})$ and that of $T 1$ (T1-2 or exon $8 \mathrm{a} / 8 \mathrm{~b})$ are relics of the ERV-MT2A and -MT2B sequences, respectively; these TE relics have developed strong and stable splice sites to be frequently recruited into 5'-UTRs of the transcripts of the respective gene (Table 1 and Figure 5). We also detected apparent T2-T1 "chimeric" transcripts that involve only 5'UTRs (Figure 4). On closer examination, all the discerned T2 and T1 5'-UTR exons, with the exception of the constitutive exon 1 and the exon 2 of $T 1$ (T1-2, or exon $7 \mathrm{a} / 7 \mathrm{~b})$, are TE remnants (Figures $2 \mathrm{~A}$ and $2 \mathrm{~B}$, see bottom TE annotations). However, we cannot rule out the possibility that the T1-2 exon was also originally derived from a TE sequence but had lost its TE features through evolution for recognition. In other words, all 5'-UTR exons, except exon 1, may, in fact, be products of exonised TE sequences recruited through alternative splicing.

Several salient features of TE insertions in the human and mouse genomes have been described based on bioinformatics analysis: (i) the TE exons are mostly intronic; (ii) all TE families can be exonised; (iii) TE exons are found mostly in the UTR, and (iv) potential tissue-specific association $[31,32]$. In this report, the depicted exon organization of the $T 1$ and $T 2$ transcripts has provided direct experimental evidence to support all of the above features of TE-derived exons. An important mechanism that contributes to the exonisation of Alu, a highly repetitive and primate-specific TE, is the RNA-editing-mediated adenosine-to-inosine (A-to-I) modification [33-35]. A-to-I RNA editing is catalysed by adenosine deaminase acting on double-stranded RNA stretches of primary transcripts formed by annealing of inverted-repeat sequences in the pre-mRNA [34]. The dense field of predominantly LINE1 and ERV sequences in the T2/T1 locus provides ample opportunities for the $T 2 / T 1$ pre-mRNA species to form double-stranded structures for adenosine deaminasemediated RNA editing. Furthermore, the TE exonization may be driven by the use of cryptic exonic splicing enhancers (ESEs) as proposed by Lin et al. [32]. The exact mechanism that is responsible for exonisation of TE sequences into the $T 1$ and $T 2$ transcripts is a subject for further investigation.

Biologically, TE insertions into 5'-UTRs of transcripts have been shown to influence gene expression at the level of transcription through the creation of new transcription factor binding sites or by other transcriptional mechanisms [36-38]. Alternatively, the presence of TE sequences could introduce deleterious uAUGs and uORFs to repress translational initiation [39-41] as we have demonstrated for selected $T 1$ transcripts (Figure 7 ). The complexity of the $T 1$ transcript population in the developing embryo impedes detailed determination of the relative abundances of the discerned transcripts.

\section{Conclusion}

This work provides evidence to indicate that exonisation of TE sequences is a frequent event in the transcription of retrogenes during embryonic development and in the testis and TE exonisation may contribute to post-transcriptional regulation of expression of retrogenes through translational repression. The T2/T1 locus, thus, provide a spatio-temporal model for further dissection of developmentally-regulated and testis-specific transcription and possible biological significance of TE exonisation of retrogenes.

\section{Methods \\ Cell lines and rats}

The rat insulinoma cell line RIN-m5F was acquired from the Bioresource Collection and Research Centre, Taiwan. Sprague Dawley rats were used throughout this work and were obtained from the Laboratory Animal Centre, National Yang-Ming University, Taiwan. This study was approved by the Institutional Animal Care and Use Committee (IACUC) of the Taipei Veterans General Hospital. The animals were sacrificed according to the IACUC guidelines.

\section{RNA preparation and expression profiling by $R T-P C R$}

Total RNAs were prepared from rat tissues using the TRIReagent $^{\circledast}$ (MRC, Cincinnati, OH) and were treated with DNase before reverse transcription. Total RNA from whole embryos from the embryonic stages E12 to E20 were purchased from Zyagen. RT-PCR-based expression profiling was performed as described $[15,20]$. Briefly, five microgram aliquots of total RNA were initially used to generate the first-strand cDNA using an oligo(dT) primer and the SuperScript ${ }^{\circledR}$ II First-Strand Synthesis System (Invtirogen). For the standard expression profiling (Figure $1 \mathrm{~B})$, the RT products were subjected to a first-round PCR using the Ex1a-B + T1SP-R1 or Ex1a-B + T2SP-R1 primer pairs (see Table 2 for primer sequences and the relative positions as depicted in Figure 1A) and the following PCR conditions using the Fast-Run ${ }^{\circledR}$ Taq Master Mix Kit (Pro- 
tech): $94^{\circ} \mathrm{C}$ for $3 \mathrm{~min}$ for initial denaturation followed by 35 cycles at $94^{\circ} \mathrm{C}$ for $30 \mathrm{sec}, 59^{\circ} \mathrm{C}$ (for $\mathrm{T} 1$ ) or $61^{\circ} \mathrm{C}$ (for T2) for $30 \mathrm{sec}, 72^{\circ} \mathrm{C}$ for $3 \mathrm{~min}$ and the reaction was further extended at $72^{\circ} \mathrm{C}$ for $10 \mathrm{~min}$ before termination of the reaction. The first-round PCR products were diluted 200fold before being used in the second-round PCR using the Ex1a-A + T1SP-R3 or Ex1a-A + T2SP-R3 primer pairs (Table 2 and Figure 1A) as described above except for 25 cycles of amplification and with an annealing temperature of $57^{\circ} \mathrm{C}$ or $51^{\circ} \mathrm{C}$ for $\mathrm{T} 1$ or $T 2$, respectively. The $\mathrm{T} 1$ extended PCR analysis (Figure $1 \mathrm{C}$ ) was performed essentially as for the standard profiling PCR as follows: For the first-round PCR, undiluted RT product was subjected to amplification using the primers Ex1a-B and 3096R (Table 2 and Figure 1A) and an annealing temperature of $61^{\circ} \mathrm{C}$ for 35 cycles. The first-round PCR products were diluted 100 times for the second-round PCR using the primers Ex1a-A and 2965R (Table 2 and Figure 1A). The PCR conditions are as in the first-round PCR except that the primer annealing temperature was at $68^{\circ} \mathrm{C}$. In all expression profiling experiments, $\beta$-actin gene-specific primers were included as an internal control in all the RT-PCR experiments. Specific discrimination between $T 1$ and $T 2$ in the expression profiling was demonstrated by the use of $T 1$ or T2 sequence-containing plasmid DNA originally derived from the testis in the previous study [20]; these plasmid controls resulted in positive bands only in the designated reactions (data not shown but a demonstration of a similar assay is shown in Figure 3A).

\section{5'- and 3'-RACE and bioinformatics analysis}

The procedure of rapid amplification of cDNA ends (RACE) was used to derive sequences of the 5'- and 3'halves of T1 and T2 mRNAs for construction of full-length transcript sequences. For the RACE experiments, a SMART $^{\circledast}$ RACE cDNA Amplification Kit (BD Biosciences) was used according to the manufacturer's instructions and as described $[1,20]$. To increase specificity and sensitivity, nested PCR was routinely performed using the Nested Universal Primer A included in the SMART kit and T1- or T2-gene specific nested primers (5'RACE-GSP-R2 or 3'RACE-GSP-F1 for 5'- or 3'-RACE, respectively, see Table $2)$. All RACE-generated sequences were cloned into the pGEM $^{\circledR}$-TEasy vector for sequence analysis. Nucleotide sequences were subjected to BLAST searches of the GenBank rat resources database [http:/ www.ncbi.nlm.nih.gov/genome/guide/rat/; assembly version RGSC v3.4, as on December 1, 2008] using default parameters and filters. The Lasergen $e^{\circledR}$ software programs

Table 2: Oligonucleotide primers used in this study

\begin{tabular}{|c|c|}
\hline Primers & Sequence $\left(5^{\prime}-3^{\prime}\right)$ \\
\hline & (A) Primers for RT-PCR expression profiling \\
\hline Exla-B & CAGGGAGACAGCTGATACATTTAG \\
\hline Exla-A & TCCAGCCAGAGAAAGACTCATCATC \\
\hline TISP-FI & CCCACTTCTTCAGAATTCCAGCAT \\
\hline TISP-RI & GTTCAAGACATCAGACAAGAATAA \\
\hline TISP-R2 & CTTTAGTCTTCAGGTTCTCTGCTC \\
\hline TISP-R3 & AACATCTGAGATCTTTGTAATAAC \\
\hline T2SP-FI & CGCACATCTTCAGGATTCTGGTCG \\
\hline T2SP-RI & AGACATCAAACAAGAATCCAACAG \\
\hline T2SP-R2 & CCCTAGTCTTCAGGTGCTCTGTTT \\
\hline T2SP-R3 & AACATTTGACATTTTTGTAATAAG \\
\hline $2965 R$ & GAGATTGCCACACAGATGATAGAG \\
\hline \multirow[t]{2}{*}{$3096 \mathrm{R}$} & TTGACCATATAAACCAACTGACAG \\
\hline & (B) 5'- and 3'-RACE gene-specific primers \\
\hline 5' RACE-GSP-RI & CACCAGGCTGCAGTCTGTGAAGAGG \\
\hline 5' RACE-GSP-R2 & CTTCACTGGCCTCTAATGAGAACC \\
\hline 3' RACE-GSP-FI & AGATTACCTGTCTGTTTACCTGGG \\
\hline \multirow[t]{2}{*}{ 3' RACE-GSP-F2 } & TTGGGCAAAGGTACAGTTCTGGAT \\
\hline & (C) Primers for cloning of 5'-UTR for uORF assays \\
\hline TI uORF-F & TTGCGTCCGGCTCTCAGC \\
\hline \multirow{2}{*}{ TI uORF-R Ncol } & AACCATGGTTTCTGGAGGTAGTTTGGA \\
\hline & (D) Primers for site-directed mutagenesis \\
\hline F215 & CCTGAAGAGTAAGCAGACCAAGAGAACATAGAG \\
\hline R247 & CTCTATGTTCTCTTGGTCTGCTTACTCTTCAGG \\
\hline F373 & GTCTATATAAGCTCAAGGAAGAAGGAAGC \\
\hline \multirow[t]{2}{*}{ R40I } & GCTTCCTTCTTCCTTGAGCTTATATAGAC \\
\hline & (E) Primers for luciferase RT-PCR \\
\hline Luc-F (I265-1288) & ACATAGCTTACTGGACGAAGACG \\
\hline Luc-R (I557-I580) & GTAAGACCTTTCGGTACTTCGTCC \\
\hline
\end{tabular}


package obtained from DNAstar ${ }^{\circledR}$ was used for in-house sequence alignment and nucleotide sequence analysis.

\section{Plasmid construction, site-specific mutagenesis, transient transfection and luciferase activity assay}

The 5'-UTR sequences of selected T1 transcripts were derived from the RNA by PCR amplification using oligo(dT)-primed RT products and reverse primers flanked with NcoI recognition sequence for cloning into the SV40 promoter-driven pGL3-Promoter and the SV40 promoter-plus-enhancer pGL3-Control luciferase reporter plasmids (Promega). For site-directed mutagenesis, oligonucleotides encompassing the mutations and containing restriction cloning sites were used as primers in PCR amplification reactions as described [42]. For transient transfection experiments, $\mathrm{CHO}-\mathrm{K} 1$ cells were seeded onto 24-well Petri dishes $24 \mathrm{~h}$ prior to transfection. Cells were co-transfected in duplicates with the luciferase constructs and the thymidine kinase promoter-driven Renilla Luciferase plasmid using the Lipofectamine Plus reagent (Life Technologies) as described [20,43]. Forty-eight hours post-transfection, cells were lysed and duplicates of $20 \mu \mathrm{l}$ aliquots of the cell lysate were removed for measurement of the luciferase activities in a luminometer using the Dual-Luciferase Reporter Assay Kit according to the user's manual (Promega).

\section{Western blot analysis}

CHO-K1 cells transfected with Luciferase reporter constructs were harvested 24, 36 and 48 h after transfection. Subsequent processing of the lysed cells for western blot analysis using an anti-luciferase antibody (Novus) was performed as previously described [42]. Signals were visualised by chemiluminescence after treating the membrane blot with a Western Lightning Plus-ECL reagent (PerkinElmer) according to the manufacturer's recommendations.

\section{Authors' contributions}

$\mathrm{CJH}$ and KBC conceived and designed all the experiments and did the data analysis in the study; WYL and CMC performed all the experiments; $\mathrm{CJH}$ and $\mathrm{KBC}$ wrote the paper.

\section{Additional material}

\section{Additional file 1}

Splice sites in the TE-associated genomic segments that contribute to the 5'-UTR of the T1 transcripts. The splice sites in the (A) L1/ERV and (B) S1-hAT TE sequences are as defined in the text. In the sequences, exons and introns are shown in upper-and lowercase letters, respectively. The 5'- and 3'-splice sites (5'- and 3'-ss) are shown. At the bottom of each sequence, the GIRI RepBase-derived tabulation of the TE sequences is also shown.

Click here for file

[http://www.biomedcentral.com/content/supplementary/14712199-10-74-S1.doc]

\section{Additional file 2}

Alignment of the rat $(\mathrm{Rn})$ and mouse $(\mathrm{Mm})$ exon 1 sequence. The $\mathrm{Mm}$ and $R n$ exon 1 sequences are the mouse and rat genomic sequences that align with the common leader exon 1 of the rat $\mathrm{T} 1$ and $\mathrm{T} 2$ transcripts. The overall identity between the mouse and rat exon 1 genomic sequences is determined to be $75.1 \%$. The figure is taken from an NCBI BLAST alignment.

Click here for file

[http://www.biomedcentral.com/content/supplementary/14712199-10-74-S2.ppt]

\section{Acknowledgements}

The authors thank Yao-Hui Tsai for technical assistance and Tsung-Sheng $\mathrm{Su}$ for discussion. This work was supported by grants $\mathrm{V} 97 \mathrm{Cl}-055$ and V $98 \mathrm{Cl}-020$ to KBC and CJH from the Taipei Veterans General Hospital, Taipei, Taiwan.

\section{References}

I. Chen HH, Liu TY, Huang CJ, Choo KB: Generation of two homologous and intronless zinc-finger protein genes, Zfp352 and Zfp353, with different expression patterns by retrotransposition. Genomics 2002, 79(I): 18-23.

2. Chen HH, Liu TY, Li H, Choo KB: Use of a common promoter by two juxtaposed and intronless mouse early embryonic genes, Rnf33 and Rnf35: implications in zygotic gene expression. Genomics 2002, 80(2): | 40-143.

3. Choo KB, Chen HH, Liu TY, Chang CP: Different modes of regulation of transcription and pre-mRNA processing of the structurally juxtaposed homologs, Rnf33 and Rnf35, in eggs and in pre-implantation embryos. Nucleic Acids Res 2002, 30(22):4836-4844.

4. Maniatis T, Tasic B: Alternative pre-mRNA splicing and proteome expansion in metazoans. Nature 2002, 4I 8(6894):236-243.

5. Kim E, Goren A, Ast G: Alternative splicing: current perspectives. Bioessays 2008, 30(I):38-47.

6. Marques AC, Dupanloup I, Vinckenbosch N, Reymond A, Kaessmann $\mathrm{H}$ : Emergence of young human genes after a burst of retroposition in primates. PLoS Biol 2005, 3(I I):e357.

7. Vinckenbosch N, Dupanloup I, Kaessmann H: Evolutionary fate of retroposed gene copies in the human genome. Proc Natl Acad Sci USA 2006, 103(9):3220-3225.

8. Kleene KC, Mulligan E, Steiger D, Donohue K, Mastrangelo MA: The mouse gene encoding the testis-specific isoform of Poly(A) binding protein (Pabp2) is an expressed retroposon: intimations that gene expression in spermatogenic cells facilitates the creation of new genes. I Mol Evol 1998, 47(3):275-28I.

9. Swanson WJ, Vacquier VD: The rapid evolution of reproductive proteins. Nat Rev Genet 2002, 3(2): I37-I44.

10. Schmidt EE: Transcriptional promiscuity in testes. Curr Biol 1996, 6(7):768-769.

II. Ma J, Svoboda P, Schultz RM, Stein P: Regulation of zygotic gene activation in the preimplantation mouse embryo: global activation and repression of gene expression. Biol Reprod 200 I, 64(6): $17|3-172|$.

12. Ko MS, Kitchen IR, Wang X, Threat TA, Hasegawa A, Sun T, Grahovac MJ, Kargul G], Lim MK, Cui Y, et al.: Large-scale cDNA analysis reveals phased gene expression patterns during preimplantation mouse development. Development 2000, I 27(8): I737- I 749.

13. Conley $A B$, Piriyapongsa J, Jordan IK: Retroviral promoters in the human genome. Bioinformatics 2008, 24(14): I563-I567.

14. Yohn CT, Jiang Z, McGrath SD, Hayden KE, Khaitovich P, Johnson ME, Eichler MY, McPherson JD, Zhao S, Paabo S, et al.: Lineage-specific expansions of retroviral insertions within the genomes of African great apes but not humans and orangutans. PLOS Biol 2005, 3(4): el I0. 
15. Huang CJ, Chen CY, Chen HH, Tsai SF, Choo KB: TDPOZ, a family of bipartite animal and plant proteins that contain the TRAF (TD) and POZ/BTB domains. Gene 2004, 324: I 17-127.

16. Zapata JM, Martinez-Garcia V, Lefebvre S: Phylogeny of the TRAF/ MATH domain. Adv Exp Med Biol 2007, 597: I-24.

17. Wajant H, Henkler F, Scheurich P: The TNF-receptor-associated factor family: scaffold molecules for cytokine receptors, kinases and their regulators. Cell Signal 200I, 13(6):389-400.

18. Bradley JR, Pober JS: Tumor necrosis factor receptor-associated factors (TRAFs). Oncogene 200I, 20(44):6482-649I.

19. Kelly KF, Daniel JM: POZ for effect - POZ-ZF transcription factors in cancer and development. Trends Cell Biol 2006, I6(II):578-587

20. Choo KB, Hsu MC, Chong KY, Huang CJ: Testis-specific expression and genomic multiplicity of the rat Rtdpoz genes that encode bipartite TRAF- and POZ/BTB-domain proteins. Gene 2007, 387(I-2): | 4 |-| 49 .

2I. Hernandez-Munoz I, Lund AH, Stoop P van der, Boutsma E, Muijrers I, Verhoeven E, Nusinow DA, Panning B, Marahrens $Y$, van Lohuizen M: Stable $X$ chromosome inactivation involves the PRCI Polycomb complex and requires histone MACROH2AI and the CULLIN3/SPOP ubiquitin E3 ligase. Proc Natl Acad Sci USA 2005, I02(2I):7635-7640.

22. Kwon JE, La M, Oh KH, Oh YM, Kim GR, Seol JH, Baek SH, Chiba T, Tanaka K, Bang OS, et al:: BTB domain-containing speckle-type POZ protein (SPOP) serves as an adaptor of Daxx for ubiquitination by Cul3-based ubiquitin ligase. J Biol Chem 2006, 28 I( I 8): | 2664-I 2672.

23. Di Marcotullio L, Ferretti E, Greco A, De Smaele E, Screpanti I, Gulino A: Multiple ubiquitin-dependent processing pathways regulate hedgehog/gli signaling: implications for cell development and tumorigenesis. Cell Cycle 2007, 6(4):390-393.

24. Zhang Q, Zhang L, Wang B, Ou CY, Chien CT, Jiang J: A hedgehoginduced BTB protein modulates hedgehog signaling by degrading Ci/Gli transcription factor. Dev Cell 2006, I0(6):719-729.

25. Choo KB, Chen HH, Cheng WT, Chang HS, Wang M: In silico mining of EST databases for novel pre-implantation embryospecific zinc finger protein genes. Mol Reprod Dev 200I, 59(3):249-255.

26. Kempken F, Windhofer F: The hAT family: a versatile transposon group common to plants, fungi, animals, and man. Chromosoma 200I, I I0(I): I-9.

27. Rubin E, Lithwick G, Levy AA: Structure and evolution of the hAT transposon superfamily. Genetics 200I, I 58(3):949-957.

28. Ko MS: Embryogenomics: developmental biology meets genomics. Trends Biotechnol 200I, I (1 12):5I|-5|8.

29. Gibbs RA, Weinstock GM, Metzker ML, Muzny DM, Sodergren El, Scherer S, Scott G, Steffen D, Worley KC, Burch PE, et al.: Genome sequence of the Brown Norway rat yields insights into mammalian evolution. Nature 2004, 428(6982):493-52I.

30. Bourque G, Leong B, Vega VB, Chen X, Lee YL, Srinivasan KG, Chew JL, Ruan $Y$, Wei CL, Ng HH, et al.: Evolution of the mammalian transcription factor binding repertoire via transposable elements. Genome Res 2008, 18(II):1752-1762.

31. Sela N, Mersch B, Gal-Mark N, Lev-Maor G, Hotz-Wagenblatt A, Ast G: Comparative analysis of transposed element insertion within human and mouse genomes reveals Alu's unique role in shaping the human transcriptome. Genome Biol 2007, 8(6): $\mathrm{R}$ /27.

32. Lin L, Jiang $P$, Shen $S$, Sato $S$, Davidson BL, Xing Y: Large-scale analysis of exonized mammalian-wide interspersed repeats in primate genomes. Hum Mol Genet 2009, I 8(I 2):2204-22I4.

33. Rueter SM, Dawson TR, Emeson RB: Regulation of alternative splicing by RNA editing. Nature 1999, 399(6731):75-80.

34. Lev-Maor G, Sorek R, Shomron N, Ast G: The birth of an alternatively spliced exon: 3 ' splice-site selection in Alu exons. Science 2003, 300(5623): |288-|29|.

35. Moller-Krull M, Zemann A, Roos C, Brosius J, Schmitz J: Beyond DNA: RNA editing and steps toward Alu exonization in primates. J Mol Biol 2008, 382(3):601-609.

36. Landry JR, Medstrand P, Mager DL: Repetitive elements in the $\mathbf{5}$ untranslated region of a human zinc-finger gene modulate transcription and translation efficiency. Genomics 200I, 76(I3): $110-116$.
37. Belancio VP, Hedges DJ, Deininger P: LINE-I RNA splicing and influences on mammalian gene expression. Nucleic Acids Res 2006, 34(5): $|5| 2-152 \mid$.

38. Deininger PL, Batzer MA: Mammalian retroelements. Genome Res 2002, I 2(10): I 455- I 465.

39. Morris DR, Geballe AP: Upstream open reading frames as regulators of mRNA translation. Mol Cell Biol 2000, 20(23):8635-8642.

40. Churbanov A, Rogozin IB, Babenko VN, Ali H, Koonin EV: Evolutionary conservation suggests a regulatory function of AUG triplets in 5'-UTRs of eukaryotic genes. Nucleic Acids Res 2005, 33(I 7):55।2-5520.

41. lacono M, Mignone F, Pesole G: UAUG and uORFs in human and rodent 5'untranslated mRNAs. Gene 2005, 349:97-105.

42. Huang C], Chang JG, Wu SC, Choo KB: Negative transcriptional modulation and silencing of the bi-exonic Rnf35 gene in the preimplantation embryo. Binding of the CCAAT-displacement protein/Cux to the untranslated exon I sequence. J Biol Chem 2005, 280(35):3068I-30688.

43. Huang C], Wu SC, Choo KB: Transcriptional modulation of the pre-implantation embryo-specific Rnf35 gene by the Y-box protein NF-Y/CBF. Biochem J 2005, 387(Pt 2):367-375.

Publish with Biomed Central and every scientist can read your work free of charge

"BioMed Central will be the most significant development for disseminating the results of biomedical research in our lifetime. "

Sir Paul Nurse, Cancer Research UK

Your research papers will be:

- available free of charge to the entire biomedical community

- peer reviewed and published immediately upon acceptance

- cited in PubMed and archived on PubMed Central

- yours - you keep the copyright 